\title{
Extracellular Signal-Regulated Kinase in the Basolateral Amygdala, but not the Nucleus Accumbens Core, is Critical for Context-Response-Cocaine Memory Reconsolidation in Rats
}

\author{
Audrey M Wells', Amy A Arguello', Xiaohu Xie', Megan A Blanton', Heather C Lasseter', \\ Andrew M Reittinger' and Rita A Fuchs*,' \\ 'Department of Psychology, University of North Carolina, Chapel Hill, NC, USA
}

\begin{abstract}
The reconsolidation of cocaine memories following retrieval is necessary for the sustained ability of a cocaine-paired environmental context to elicit cocaine seeking. Extracellular signal-regulated kinase (ERK) is an intracellular signaling molecule involved in nucleus accumbens core (NACc)-mediated reconsolidation of Pavlovian cocaine memories. Here, we used a rodent model of drug contextelicited relapse to test the hypothesis that ERK would be similarly required for the reconsolidation of context-response-cocaine memories that underlie drug context-induced reinstatement of instrumental cocaine-seeking behavior, with a focus on the NACc and on the basolateral amygdala (BLA), another important locus for the reconsolidation of cocaine memories. We show that the mitogenactivated protein kinase (MEK)/ERKI/2 inhibitor, U0I26 (I.0 $\mu \mathrm{g} / 0.5 \mu \mathrm{l} /$ hemisphere), microinfused bilaterally into the BLA—but not the $\mathrm{NACc}$-immediately after brief re-exposure to a previously cocaine-paired context (that is, cocaine-memory reactivation), significantly attenuated subsequent drug context-induced cocaine seeking relative to vehicle (VEH). This effect in the BLA was associated with a transient inhibition of ERKI/2 phosphorylation, and it depended on memory reactivation given that U0I26 administered following exposure to a novel context did not alter subsequent cocaine seeking. Furthermore, similar to U0 I26, baclofen + muscimol-induced ( $\mathrm{B}+\mathrm{M} ; \quad$ 106.8/5.7 $\mathrm{ng} / 0.5 \mu \mathrm{l} /$ hemisphere) neural inactivation of the NACc, following cocaine-memory reactivation, failed to alter subsequent cocaine seeking. These findings demonstrate that ERK activation in the BLA, but not the NACc, is required for the reconsolidation of context-response-cocaine associative memories. Together with prior research, these results suggest that contextual drug-memory reconsolidation in Pavlovian and instrumental settings involves distinct neuroanatomical mechanisms.

Neuropsychopharmacology (2013) 38, 753-762; doi:I0.1038/npp.2012.238; published online 12 December 2012
\end{abstract}

Keywords: memory reconsolidation; basolateral amygdala; nucleus accumbens core; reinstatement; extracellular signal-regulated kinase; cocaine

\section{INTRODUCTION}

Exposure to cocaine-associated environmental contexts reliably elicits craving and relapse in abstinent cocaine addicts (Childress et al, 1988). This phenomenon requires the maintenance of cocaine-related associative memories in long-term memory (LTM) stores (Crombag et al, 2008; Fuchs et al, 2008b). The memory reconsolidation hypothesis posits that memory traces are rendered labile upon retrieval and must undergo a protein synthesis-dependent reconsolidation process to subsist (Finnie and Nader 2012).

\footnotetext{
* Correspondence: Dr RA Fuchs, Department of Psychology, University of North Carolina at Chapel Hill, CB\# 3270, Davie Hall, Room I21, Chapel Hill, NC 27599-3270, USA, Tel: +919843 9112,

Fax: +919962 2537,E-mail: rfuchs@unc.edu

Received 17 July 2012; revised 9 November 2012; accepted II November 2012; accepted article preview online 21 November 2012
}

Promising findings from human studies and animal models of drug relapse suggest that interfering with the reconsolidation of maladaptive drug-related memories can reduce their impact on future drug-seeking behavior (for review, see Sorg, 2012). Hence, elucidating the molecular mechanisms of cocaine-memory reconsolidation will be invaluable for the development of highly selective pharmacotherapies aimed at cocaine relapse prevention.

Phosphorylation-dependent activation of the mitogenactivated protein kinase, ERK, (Derkinderen et al, 1999) likely contributes to the reconsolidation of maladaptive cocaine memories. In support of this, inputs from extracellular and upstream intracellular signals important for drug-memory reconsolidation, including the stimulation of $\beta$-adrenergic (Milton et al, 2008b; Fricks-Gleason and Marshall, 2008) and N-methyl aspartate receptors (Brown et al, 2008; Milton et al, 2008a), as well as the activation of protein kinase A (Sanchez et al, 2010), are integrated at the 
level of ERK activation in the cytoplasm of neurons (Sweatt, 2001). In turn, ERK phosphorylates several transcription factors (Goodman, 1990; Impey et al, 1999; Sgambato et al, 1998) that have also been implicated in drug-memory reconsolidation, including the cAMP response element binding protein (CREB) and $\mathrm{E}$ twenty-six (ETS)-like transcription factor 1 (Miller and Marshall, 2005). Furthermore, evidence suggests that ERK itself is required for the reconsolidation of object recognition memories (Kelly et al, 2003; Silingardi et al, 2011), conditioned-fear associations (Duvarci et al, 2005), and importantly, Pavlovian cocainerelated memories that underlie the expression of cocaineconditioned place preference (CPP; Miller and Marshall 2005; Valjent et al, 2006). However, the role of ERK in the reconsolidation of contextual drug memories has not been assessed in an instrumental paradigm. Hence, the present study utilized the extinction-reinstatement model of environmentally triggered drug relapse in order to determine whether ERK signaling would be required for the reconsolidation of context-response-cocaine memories in two brain regions that critically regulate drug-memory reconsolidation: the basolateral amygdala (BLA; Sanchez et al, 2010; Li et al, 2010; Fuchs et al, 2009) and the nucleus accumbens core (NACc; Miller and Marshall, 2005; Théberge et al, 2010).

\section{MATERIALS AND METHODS}

\section{Animals}

Male Sprague-Dawley rats $(N=98$, Charles-River, Wilmington, MA) were housed in a temperature-controlled vivarium on a reversed light-dark cycle. Rats were maintained on 20-25 $\mathrm{g}$ of rat chow per day. Water was available ad libitum. The housing and treatment of animals followed the 'Guide for the Care and Use of Laboratory Rats' (Institute of Laboratory Animal Resources on Life Sciences, National Research Council, 2011) and were approved by the Institutional Animal Care and Use Committee of the University of North Carolina at Chapel Hill.

\section{Food Training and Surgery}

To expedite cocaine self-administration training, rats were first trained to lever press under a fixed-ratio 1 (FR-1) schedule of food reinforcement overnight. Forty-eight hours later, they were surgically implanted with intravenous jugular catheters and twenty-six gauge stainless steel guide cannulae (Plastics One) aimed bilaterally either at the BLA $(-2.7 \mathrm{~mm} \mathrm{AP}, \pm 5.1 \mathrm{~mm} \mathrm{ML},-6.7 \mathrm{~mm} \mathrm{DV}$, relative to bregma) or the NACc (angled laterally by $10^{\circ} ;+1.4 \mathrm{~mm} \mathrm{AP}$, $\pm 3.1 \mathrm{ML},-4.8 \mathrm{~mm} \mathrm{DV}$, relative to bregma). The food training, surgery, and post-operative care procedures have been described previously (Fuchs et al, 2009).

\section{Self-Administration and Extinction Training}

After surgical recovery, daily 2 -h self-administration training sessions were conducted in standard operant conditioning chambers that were configured to one of two distinctly different contexts (Contexts 1 and 2, described in Supplementary Table 1) during the rats' dark cycle. Active lever presses resulted in cocaine reinforcement (cocaine hydrochloride; $0.15 \mathrm{mg} / 0.05 \mathrm{ml}$ per infusion, i.v.; NIDA, Research Triangle Park, NC) under a FR-1/time-out-20 s schedule, as described previously (Fuchs et al, 2009). Responses on the other (inactive) lever were recorded but had no scheduled consequences. Training continued until rats reached the acquisition criterion (ie, minimum 10 sessions with $\geqslant 10$ cocaine infusions/session).

Next, rats received seven daily 2 -h extinction-training sessions in the context ( 1 or 2 ) that had not been used for self-administration. During extinction training, active and inactive lever responses were recorded but had no scheduled consequences. Following the fourth extinctiontraining session, rats were adapted to the intracranial microinfusion procedure, as described previously (Fuchs et al, 2009).

Experiment 1a. Experiment 1a evaluated whether intraBLA administration of the MEK/ERK inhibitor, U0126, which prevents the phosphorylation-dependent activation of ERK (London and Clayton, 2008), would impair instrumental, context-response-cocaine memory reconsolidation. On the day after the last extinction training session, rats were re-exposed to the cocaine-paired context for $15 \mathrm{~min}$ to initiate the destabilization and reconsolidation of cocaine memories (ie, cocaine-memory reactivation; Fuchs et al, 2009; see Figure 1a). The levers were extended but cocaine was not infused upon lever pressing given that cocaine itself stimulates ERK phosphorylation (Zhai et al, 2008). Immediately after the session, rats received bilateral intra-BLA microinfusions of 5\% DMSO/6\% TWEEN vehicle (VEH) or U0126 $(1.0 \mu \mathrm{g} / 0.5 \mu \mathrm{l} /$ hemisphere, based on Miller and Marshall, 2005). Treatment assignment was counterbalanced based on previous cocaine intake. Infusions were delivered over $2 \mathrm{~min}$, and the injection cannulae were left in place for $1 \mathrm{~min}$ before and after the microinfusion.

\section{Post-Reactivation Extinction and Test of Drug Context- Induced Cocaine Seeking}

Following memory reactivation, rats received additional daily 2 -h extinction-training sessions ( $2.28 \pm 0.20$ days) until they reached the extinction criterion (ie, $\leqslant 25$ active lever responses per session on a minimum of 2 consecutive days). Twenty-four hours later, rats were placed into the cocaine-paired context for a 2-h test of drug contextinduced cocaine seeking. Lever presses were recorded, but had no scheduled consequences. Non-reinforced active lever presses provided an index of motivation to obtain cocaine reinforcement.

Experiment $1 b$. Experiment $1 \mathrm{~b}$ examined whether the effects of intra-BLA U0126 would depend on explicit memory reactivation, consistent with genuine memory reconsolidation impairment (Nader et al, 2000b). The experimental protocols were identical to those in Experiment 1a (see Figure 1a) except that rats were placed into a novel, unpaired context (described in Supplementary Table 1) that distinctly differed from contexts 1 and 2, for $15 \mathrm{~min}$ before receiving bilateral microinfusions of $\mathrm{VEH}$ or U0126 into the BLA. Novel context exposure was expected 
a

Cocaine-memory reactivation

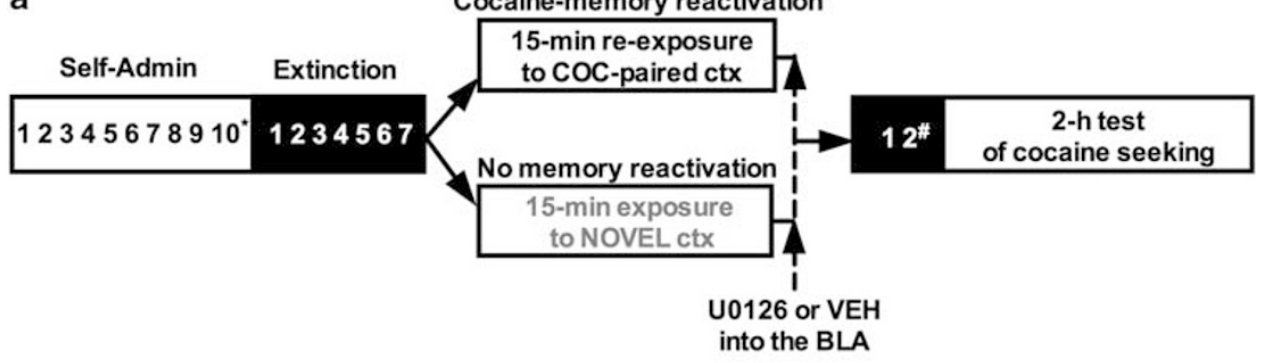

b
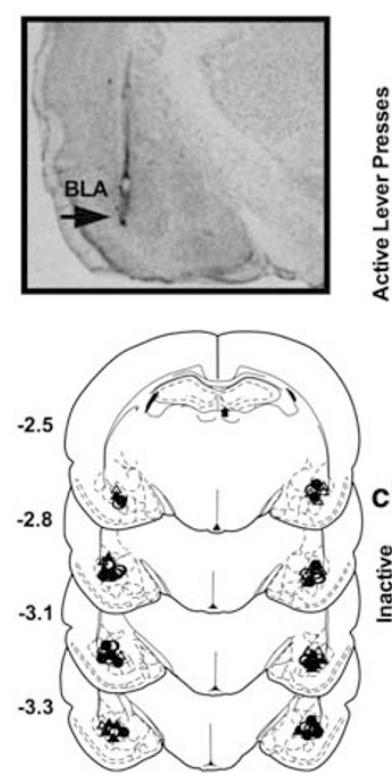

C
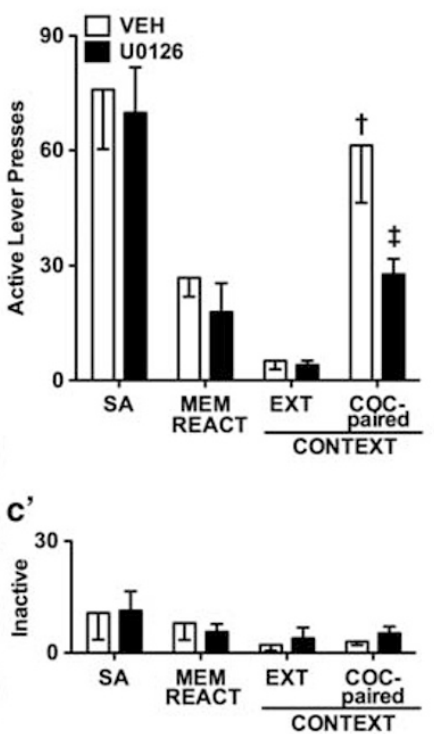

d NOVEL ctx Exposure
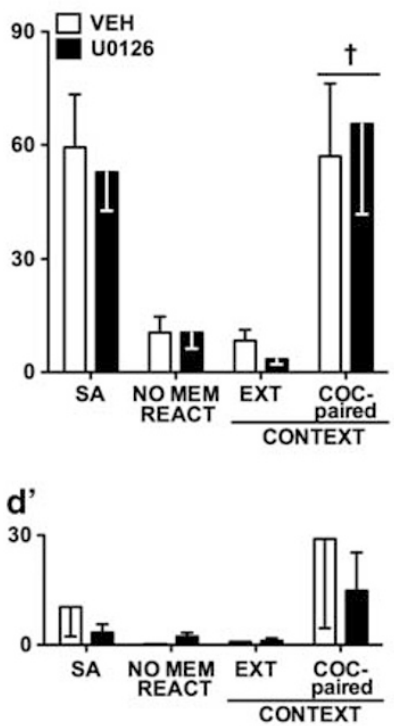

Figure I ERK inhibition in the BLA immediately after cocaine-memory reactivation impairs subsequent drug context-induced cocaine seeking, relative to VEH treatment. (a) Schematic depicting the timeline for Experiments Ia and Ib. Cocaine self-administration (SA) sessions took place in a distinct environmental context until rats reached the acquisition criterion (" 10 infusions/session for a minimum of 10 sessions). Extinction training occurred in a distinctly different context. After extinction training, rats were re-exposed to the cocaine-paired context (Cocaine-memory reactivation) for 15 min to destabilize cocaine memories and stimulate memory reconsolidation (Experiment Ia), or they were exposed to a novel, unpaired context (No memory reactivation), to provide similar experience without explicit cocaine-memory reactivation (Experiment Ib). Immediately after the session, rats received bilateral microinfusions of the MEK/ERK inhibitor, U0I26 (I.0 $\mu \mathrm{g} / 0.5 \mu \mathrm{l} /$ hemisphere) or 5\% DMSO/6\% TWEEN vehicle (VEH; $0.5 \mu / /$ hemisphere) into the BLA. Next, groups underwent additional extinction training until they met the extinction criterion $(* 25$ non-reinforced active lever responses/session for two consecutive sessions). Cocaine seeking (non-reinforced active lever responding) was then assessed in the cocaine-paired context (COC-paired). (b) Photomicrograph and schematics depicting cannula placement. The symbols denote the most ventral point of the injector cannula tracts for rats in Experiment la that received bilateral vehicle ( $\mathrm{VEH} ; n=7$, white circles) or $\mathrm{UOI} 26$ ( $n=8$, black circles) infusions into the BLA following cocaine memory reactivation and for rats in Experiment I $b$ that received bilateral VEH $(n=7$, white triangles) or $\mathrm{U} 0 \mathrm{I} 26$ ( $n=8$, black triangles) infusions following exposure to a novel, unpaired context. Numbers indicate the distance from bregma in $\mathrm{mm}$, according to the rat brain atlas of Paxinos and Watson (1997). (c) Mean $( \pm$ SEM) active lever responses during SA (mean of last three training sessions), the cocaine-memory reactivation session (MEM REACT), and during the tests of cocaine seeking in the extinction (EXT; the last session before the test in the COC-paired context) and in the COC-paired context for rats in Experiment Ia. $\left(c^{\prime}\right)$ Mean ( \pm SEM) inactive lever responses in Experiment Ia. (d) Mean ( \pm SEM) active lever responses during SA, the novel-context no memory reactivation session (NO MEM REACT), and during the tests of cocaine seeking in the EXT and COC-paired contexts in Experiment Ib. ( $\left.d^{\prime}\right)$ Mean $( \pm$ SEM) inactive lever responses in Experiment $\mathrm{l} b$. $\dagger$ denotes significant difference relative to responding in the extinction context (ANOVA context main and simple main effects, $P<0.05$ ). $\ddagger$ denotes significant difference relative to the respective $V E H$ treatment (ANOVA treatment simple main effect, $P<0.05$ ).

to circumvent explicit reactivation of the context-responsecocaine memory trace while producing similar behavioral history to that in Experiment 1a (ie, transportation to the testing room; novelty akin to that experienced by the groups in Experiment 1a that were re-exposed to the cocaine-paired context $\sim 7$ days after self-administration training).

Experiment 1c. Experiment 1c was designed to confirm that U0126 transiently suppressed the ERK1/2 activation.
The experimental protocols were identical to those in Experiment $1 \mathrm{a} / \mathrm{b}$ (see Figures $1 \mathrm{a}$ and $2 \mathrm{a}$ ) except that rats were exposed to the cocaine-paired or the novel, unpaired context for $15 \mathrm{~min}$ or they stayed in their home cages, prior to receiving bilateral microinfusions of VEH or U0126 into the BLA (see Figure 2a). The rats were euthanized $30 \mathrm{~min}$ later based on the phosphorylation kinetics of ERK1/2 (Valjent et al, 2000; Choe and McGinty, 2001; Zhang et al, 2004; Miller and Marshall, 2005) in order to capture maximal ERK phosphorylation related to the onset of 


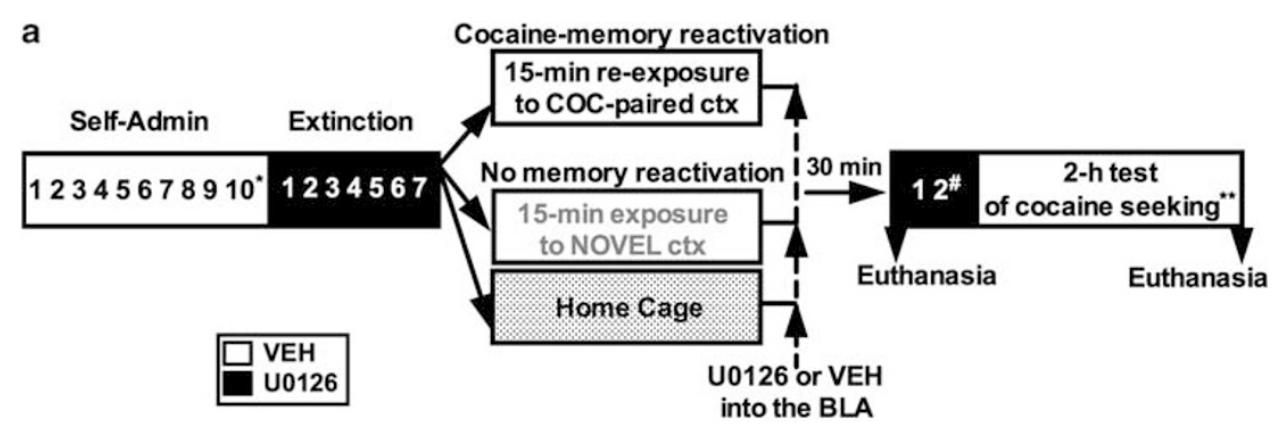

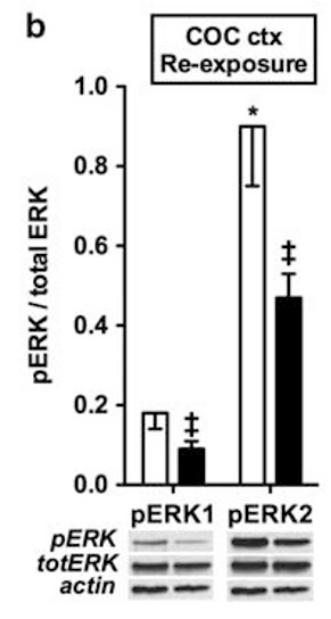

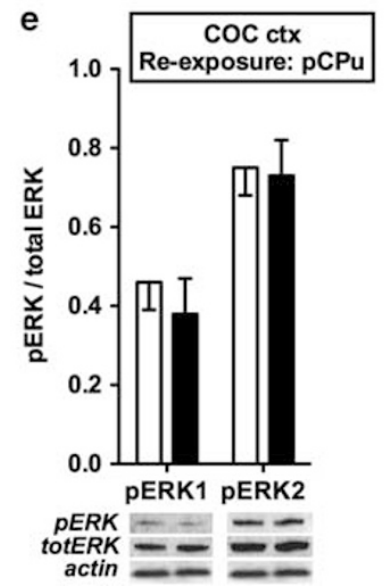

C

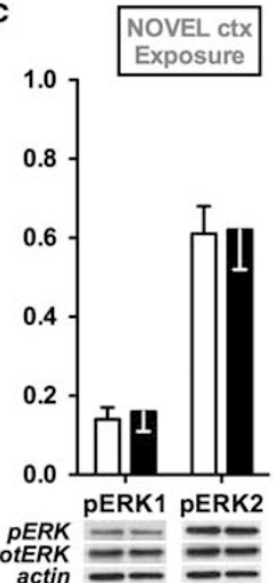

f

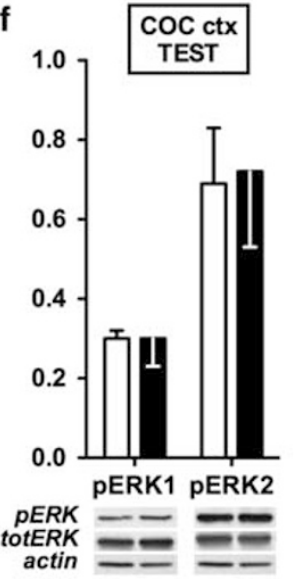

d

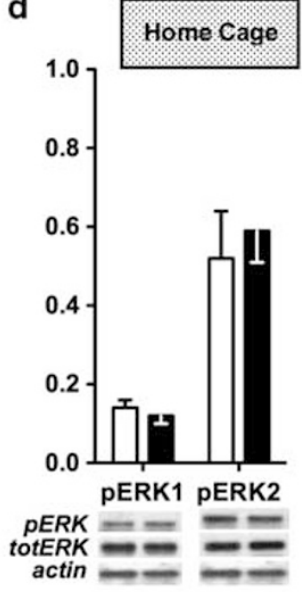

g

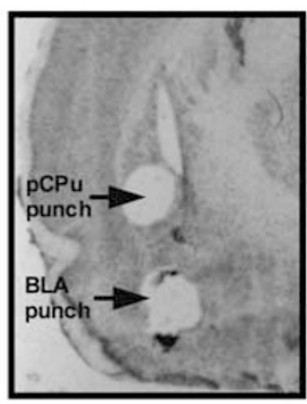

Figure 2 UOI26 in the BLA reduces ERKI and ERK2 phosphorylation in a transient and reactivation-dependent manner. (a) Schematic depicting the timeline for Experiment Ic. Experimental parameters were identical to those in Experiment Ia, except that rats received bilateral microinfusions of $\cup 0$ I 26 ( $1.0 \mu \mathrm{g} / 0.5 \mu \mathrm{l} /$ hemisphere) or VEH into the BLA following exposure to the cocaine-paired context (Cocaine-memory reactivation), the novel, unpaired context (No memory reactivation), or the home cage (No memory reactivation), and were sacrificed 30 min later. ${ }^{* *}$ Rats from Experiment la were sacrificed immediately after the test of drug context-induced cocaine seeking. (b) Mean ( \pm SEM) ERKI/2 activation (expressed as the ratio of pERK to total ERK protein levels, normalized to actin) in rats sacrificed $30 \mathrm{~min}$ after cocaine-memory reactivation and intracranial manipulations. Representative bands of pERKI, pERK2, total ERKI, total ERK2, and actin (ie, loading control) are also provided. (c) Mean ( \pm SEM) ERKI/2 activation and representative bands for rats sacrificed $30 \mathrm{~min}$ after novel context exposure and intracranial manipulations. (d) Mean ( \pm SEM) ERKI/2 activation and representative bands for home cage controls sacrificed 30 min after intracranial manipulations. (e) Mean ( \pm SEM) ERKI/2 activation in the posterior caudate putamen (pCPu; anatomical control region) and representative bands for rats sacrificed 30 min after cocaine-memory reactivation and intracranial manipulations. ( $f$ ) Mean ( \pm SEM) ERKI/2 activation and representative bands for rats sacrificed immediately after the test of cocaine seeking in Experiment Ia. (g) Photomicrograph depicting the location of BLA and PCPu tissue punches that were used for western blotting. * denotes significant difference relative to the respective home cage controls (planned, one-tail $t$-test, $P<0.05)$. $\ddagger$ denotes significant difference relative to VEH treatment (Student's $t$-test, $P<0.05$ ).

memory reconsolidation (Pedreira and Maldonado 2003). These rats were compared to rats euthanized after the final test session (ie, $\sim 72 \mathrm{~h}$ after treatment) in Experiment $1 \mathrm{a}$. Following euthanasia by rapid decapitation, brains were removed, flash frozen in isopentane, and stored at $-80^{\circ} \mathrm{C}$. Punches were taken from the BLA or the dorsally adjacent posterior caudate putamen ( $\mathrm{pCPu}$; ie, anatomical control) with $19 \mathrm{Ga}$ neuropunches (Fine Science Tools) from $40 \mu \mathrm{m}$ 
tissue sections, which were also collected to verify cannula placement. Punched tissue was stored at $-80^{\circ} \mathrm{C}$ in lysis buffer containing $10 \mathrm{mM}$ HEPES, $1 \%$ SDS, and $1 \times$ protease and phosphatase inhibitor cocktails (Sigma Aldrich, St Louis, MO).

\section{Western Blotting}

Samples were thawed on ice, manually homogenized, and boiled for $10 \mathrm{~min}$ at $100^{\circ} \mathrm{C}$. Protein concentrations were determined using the Biorad DC protein assay. For each sample, $15 \mu \mathrm{g}$ of protein were electrophoresed on a $12 \%$ Tris-HCL polyacrylamide gel and transferred to a polyvinylidene difluoride membrane for $1 \mathrm{~h}$ at $100 \mathrm{~V}$. Membranes were then blocked in 5\% milk for $1 \mathrm{~h}$ and incubated in rabbit anti-phosphorylated ERK1/2 (pERK1/2; 44/42 $\mathrm{kDA}$, respectively) $(1: 2000$, Cell Signaling, Beverly, MA) overnight $(16-20 \mathrm{~h})$ at $4{ }^{\circ} \mathrm{C}$. Membranes were then incubated in horseradish peroxidase-conjugated secondary antibody (1:10000, GE Healthcare, Piscataway, NJ), for $1 \mathrm{~h}$, followed by development with an enhanced chemiluminescence (ECL) system (Pierce Biotech, Rockford, IL). Membranes were subsequently incubated with stripping buffer (62.5 mM Tris-HCL at pH 6.7, 2\% SDS, $100 \mathrm{mM}$ betamercaptoethanol) to permit re-probing with antibodies to total (phosphorylated plus unphosphorylated) ERK1/2 $(1: 2000$, Cell Signaling, Beverly, MA) and later actin (ie, loading control; 1:50 000, Santa Cruz Biotechnology, Santa Cruz, CA). Protein levels of pERK1/2, total ERK1/2, and actin were quantified by densitometry, using NIH Image J software. To evaluate changes in ERK activation, pERK1/2 was normalized to total ERK1/2 and actin. Subsequent discussion of ERK1/2 activation refers to normalized levels.

Experiment 2a. Experiment $2 \mathrm{a}$ assessed whether intraNACc administration of U0126 would impair cocainememory reconsolidation. The experimental parameters used were identical to those in Experiment 1a, except that rats received bilateral microinfusions of VEH or U0126 into the NACc immediately after cocaine-memory reactivation (see Figure 3a).

Experiment $2 b$. Experiment $2 \mathrm{~b}$ investigated whether global neural inactivation of the NACc would impede cocaine-memory reconsolidation, in the event that ERK inhibition had no effect. The procedures used were identical to those in Experiment 2a, except that rats received bilateral intra-NACc microinfusions of phosphate buffered saline VEH or the $\mathrm{GABA}_{\mathrm{A} / \mathrm{B}}$ agonist cocktail, baclofen + muscimol (B $+\mathrm{M} ; 106.8 / 5.7 \mathrm{ng} / 0.5 \mu \mathrm{l} /$ hemisphere), immediately after cocaine memory reactivation (see Figure 3a). B + M suppresses neuronal activity while sparing fibers of passage (Martin and Ghez, 1999).

\section{Histology}

In Experiments $1 \mathrm{a}, 1 \mathrm{~b}, 2 \mathrm{a}$, and $2 \mathrm{~b}$, rats were overdosed with ketamine hydrochloride and xylazine $(66.6$ and $1.3 \mathrm{mg} / \mathrm{kg}$, i.v. or 199.8 and $3.9 \mathrm{mg} / \mathrm{kg}$, i.p., respectively, depending on catheter patency) and transcardially perfused, as described previously (Fuchs et al, 2009). Brains were dissected out and stored in $10 \%$ formaldehyde solution until they were sectioned coronally using a vibratome. Cannula placements were verified on $75 \mu \mathrm{m}$ sections stained using cresyl violet (Kodak, Rochester, NY, USA). The most ventral portion of each cannula tract was mapped onto schematics from the rat brain atlas (Paxinos and Watson, 1997).

\section{Data Analysis}

Analyses of variance (ANOVAs) or $t$-tests were conducted to examine pre-existing differences in responding during self-administration, extinction, and the memory reactivation session, as well as ERK1/2 activation and responding during the test sessions in the extinction and cocaine-paired contexts, as described in detail in Supplementary Table 2. Tukey's tests were used to probe significant main and interaction effects. Alpha was set at 0.05 .

\section{RESULTS}

\section{Histology}

Schematics and photomicrographs illustrating cannula placements are included in Figures $1 \mathrm{~b}$ and $3 \mathrm{~b}$, and Supplementary Figure 1. The target brain regions were defined as the lateral and basolateral nuclei of the amygdala and the nucleus accumbens core. High power microscopy did not reveal tissue damage (ie, extensive cell loss or gliosis). Only data from rats with correct cannula placements were included in statistical analyses.

\section{Behavioral History}

There were no pre-existing differences between the groups in cocaine intake, active or inactive lever responding during cocaine self-administration training, extinction training, or the memory reactivation session; or in the number of days required to reach the extinction criterion. These descriptive statistics are provided in Supplementary Table 3.

Experiment 1a: Effects of U0126 microinfused into the BLA following cocaine-memory reactivation on subsequent drug context-induced cocaine seeking. U0126 administration into the BLA following cocaine-memory reactivation altered subsequent cocaine seeking in a context-dependent fashion (Figure 1c; ANOVA context $\mathrm{x}$ treatment interaction, $F_{(1,13)}=5.15, P=0.03$; context main effect, $F_{(1,13)}=33.54, P<0.001$; treatment main effect, $\left.F_{(1,13)}=5.01, P=0.04\right)$. In the group that had received VEH into the BLA following cocaine-memory reactivation, active lever responding increased upon exposure to the cocaine-paired context at test, relative to that in the extinction context (Tukey's test, $P<0.05$ ). Conversely, the group that had received U0126 responded less on the active lever in the cocaine-paired context, but not the extinction context, relative to the VEH-treated group (Tukey's test, $P<0.05$ ). As a result, the U0126-treated group exhibited no difference in active lever responding in the extinction and cocaine-paired contexts.

Experiment 1b: No reactivation control experiment. U0126 administration into the BLA following exposure to a novel, unpaired context failed to alter subsequent drug 
a Self-Admin Extinction Cocaine-memory reactivation

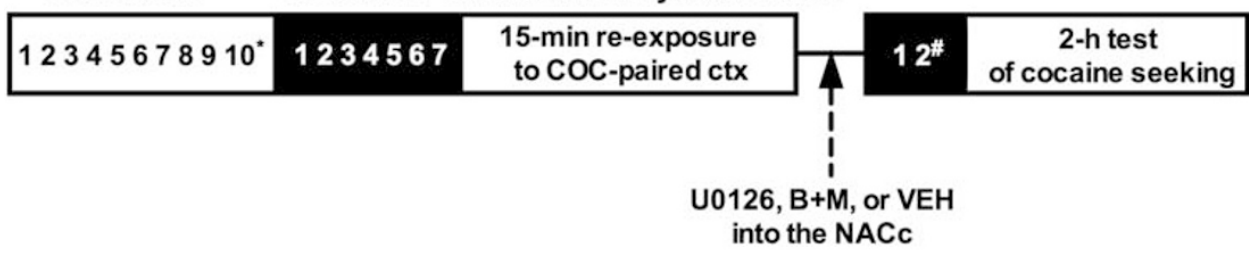

b
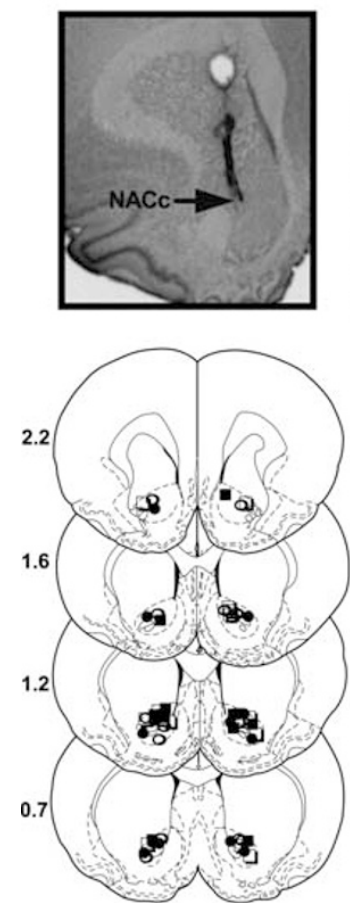

c
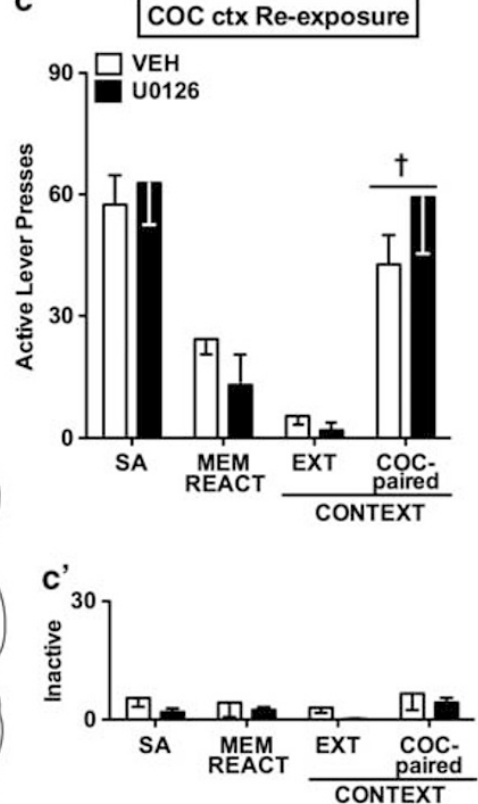

d COC ctx Re-exposure

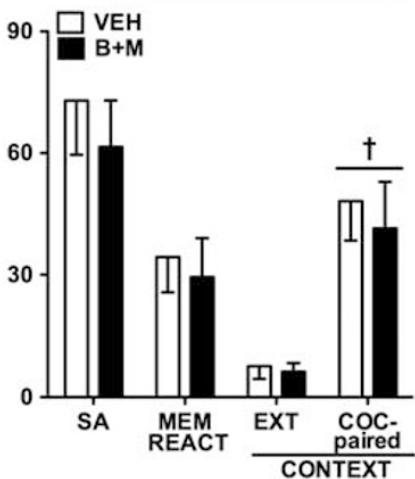

d'

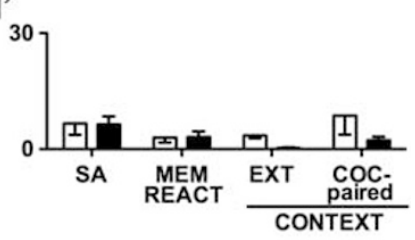

Figure 3 ERK inhibition in, or neuronal inactivation of, the NACc after cocaine-memory reactivation fails to disrupt subsequent drug context-induced cocaine seeking. (a) Schematic depicting the timeline for Experiments $2 \mathrm{a}$ and $2 \mathrm{~b}$. Experimental parameters were identical to those in Experiment Ia, except that rats in Experiment 2a received bilateral $\mathrm{U} 0 \mathrm{I} 26(\mathrm{I} .0 \mu \mathrm{g} / 0.5 \mu \mathrm{l})$ or $\mathrm{VEH}$ infusions into the NACc following cocaine-memory reactivation, and rats in Experiment $2 \mathrm{~b}$ received bilateral $\mathrm{GABA}_{\mathrm{A} / \mathrm{B}}$ agonist cocktail baclofen + muscimol $(\mathrm{B}+\mathrm{M}$; $106.8 / 5.7 \mathrm{ng} / 0.5 \mu \mathrm{l} /$ hemisphere) or phosphate buffered saline vehicle (VEH) infusions into the NACc following cocaine-memory reactivation. (b) Photomicrographic and schematic depiction of cannula placements. The symbols denote the most ventral point of the injector cannula tracts for rats that received bilateral $\mathrm{VEH}(n=7$, open circles) or $\cup 0$ I 26 ( $n=7$, filled circles) infusions into the NACc in Experiment $2 a$ and for rats that received bilateral phosphate buffered saline VEH $(n=7$, open squares) or B $+M$ ( $n=6$, filled squares) infusions in Experiment 2b. Numbers indicate the distance from bregma in mm, according to the rat brain atlas of Paxinos and Watson (I997). (c) Mean ( \pm SEM) active lever responses during self-administration (SA; mean of last three training sessions), during the I5-min cocaine-memory reactivation session (MEM REACT), and during the tests of cocaine seeking in the extinction (EXT; the last session before the test in the COC-paired context) and cocaine-paired (COC-paired) contexts in Experiment 2a. ( $\left.c^{\prime}\right)$ Mean ( \pm SEM) inactive lever responses in Experiment 2a. (d) Mean ( \pm SEM) active lever responses in Experiment 2b. $\left(d^{\prime}\right)$ Mean $( \pm$ SEM) inactive lever responses in Experiment $2 b$. $\dagger$ denotes significant difference relative to responding in the extinction context (ANOVA context main effect, $P<0.05$ ).

context-induced cocaine seeking (Figure 1d). Active lever responding increased upon exposure to the cocaine-paired context at test, relative to that in the extinction context (ANOVA context main effect only, $F_{(1,13)}=13.15, P=0.03$ ). Furthermore, there was no difference between the VEH- and U0126-treated groups in active lever responding in either context (treatment main and interaction effects, $\left.F_{(1,13)}=0.01-0.20, P=0.66-0.91\right)$.

Experiment 1c: Effects of U0126 in the BLA on ERK1/2 activation. Quantitative western blot analyses indicated that U0126 administration into the BLA significantly reduced ERK1 and ERK2 phosphorylation in the BLA in a transient and memory reactivation-dependent manner. Specifically, bilateral microinfusions of U0126 following explicit cocaine-memory reactivation significantly attenuated ERK1 $\left(t_{(13)}=3.095, P=0.009\right)$ and ERK2 $\left(t_{(13)}=3.062\right.$, $P=0.009$ ) activation in the BLA, relative to VEH (Figure $2 \mathrm{~b}$ ) when assessed $30 \mathrm{~min}$ post treatment. Conversely, when U0126 treatment was administered following exposure to the novel, unpaired context (Figure 2c) or the home cage (Figure 2d), it did not alter ERK1 $\left(t_{(12)}=0.476, P=0.64\right.$; $t_{(9)}=0.505, P=0.63$, respectively) or ERK2 $\left(t_{(12)}=0.165\right.$, $P=0.87 ; t_{(9)}=0.960, P=0.36$, respectively) activation in the BLA, relative to VEH.

Separate one-way ANOVAs indicated a marginal difference in ERK2 $\left(F_{(2,17)}=3.25 P=0.06\right)$, but not in ERK1 
$\left(F_{(2,17)}=1.29, P=0.30\right)$, activation between the groups that received $\mathrm{VEH}$ after exposure to the cocaine-paired context, the novel context, or the home cage. Furthermore, planned pairwise comparisons revealed that there was an increase in ERK2, but not ERK1, activation in these groups following exposure to the cocaine-paired context (one-tail $t_{(10)}=2.042, P=0.03$ ), but not the novel context (one-tail $\left.t_{(12)}=0.675, P=0.26\right)$, relative to the home cage.

The effects of intra-BLA U0126 administration on ERK1/2 activation were anatomically selective and transient. IntraBLA infusions of U0126 following cocaine-memory reactivation did not alter ERK1 $\left(t_{(12)}=0.673, P=0.51\right)$ or ERK2 $\left(t_{(12)}=0.196, P=0.84\right)$ activation in the $\mathrm{pCPu}$, relative to VEH (Figure 2e). Furthermore, U0126 administered following cocaine-memory reactivation did not alter ERK1 $\left(t_{(12)}=0.073, P=0.94\right)$ or ERK2 $\left(t_{(12)}=0.160, P=0.88\right)$ activation in the BLA, relative to $\mathrm{VEH}$ when assessed $\sim 72 \mathrm{~h}$ post treatment, immediately after the test of cocaine seeking (Figure $2 \mathrm{f}$ ).

Experiment 2a: Effects of U0126 microinfused into the NACc following cocaine-memory reactivation on subsequent drug context-induced cocaine seeking. U0126 administration into the NACc after cocaine-memory reactivation did not alter subsequent drug context-induced cocaine seeking (Figure 3c). Active lever responding increased upon exposure to the cocaine-paired context at test, relative to that in the extinction context (ANOVA context main effect only, $\left.F_{(1,12)}=35.88, P<0.001\right)$. Furthermore, there was no difference between the previously VEHand U0126-treated groups in active lever responding in either context (treatment main and interaction effects, $\left.F_{(1,12)}=0.67-1.64, P=0.22-0.43\right)$.

Experiment 2b: Effects of NACc neural inactivation following cocaine-memory reactivation on subsequent drug context-induced cocaine seeking. $\mathrm{B}+\mathrm{M}$ administration into the NACc following cocaine-memory reactivation failed to alter subsequent drug context-induced cocaine seeking (Figure 3d). Active lever responding increased upon exposure to the cocaine-paired context at test, relative to that in the extinction context (context main effect only, $\left.F_{(1,11)}=20.43, P=0.001\right)$. Moreover, there was no difference between the previously VEH- and $\mathrm{B}+\mathrm{M}$-treated groups in active lever responding in either context (treatment main and interaction effects, $\left.F_{(1,11)}=0.10-0.30, P=0.59-0.75\right)$.

Inactive lever responding. Inactive lever responding was low in all experiments and did not significantly differ as a function of treatment group or testing context (all Fs $<3.017$, Ps $>0.108$; Figures 1, $3 c^{\prime}$ and $d^{\prime}$ ).

\section{DISCUSSION}

Results from the present study suggest that the BLA, but not the NACc, critically regulates the reconsolidation of contextual memories that trigger instrumental cocaineseeking behavior. Consistent with this, in the present study, ERK inhibition in the BLA following brief re-exposure to the cocaine-paired context, a manipulation designed to reactivate context-response-cocaine associative memories and prompt their reconsolidation, disrupted subsequent drug context-induced cocaine-seeking behavior, presumably by inhibiting memory reconsolidation (Figure 1c). Conversely, ERK inhibition in, or GABA agonist-induced neural inactivation of, the NACc was without effect under the same experimental conditions (Figure $3 c$ and $d$ ).

\section{ERK Signaling in the BLA Critically Regulates the Reconsolidation of Contextual Cocaine Memories}

Administration of the MEK/ERK inhibitor, U0126, into the BLA following cocaine-memory reactivation decreased ERK1/2 phosphorylation in the BLA during the putative time of memory restabilization (Figure $2 \mathrm{~b}$ ) and attenuated drug context-induced cocaine seeking $\sim 72 \mathrm{~h}$ later (Figure 1b), relative to VEH. As expected from a genuine memory reconsolidation deficit, these effects depended on explicit cocaine-memory reactivation in that U0126 administration in the absence of cocaine-paired context re-exposure (ie, following novel context exposure) failed to alter subsequent drug context-induced cocaine-seeking behavior (Figure 1d). There was an increase in ERK2, but not ERK1, phosphorylation in the BLA following exposure to the cocaine-paired context, relative to the home cage (Figure 2b and d). This differential response in ERK1 and ERK 2 activation might reflect functional differences between these ERK isoforms in the effects of drugs of abuse (Mazzucchelli et al, 2002; Girault et al, 2007). Conversely, there was no increase in ERK1/2 phosphorylation following exposure to the novel context, relative to the home cage (Figure $2 c$ and $d$ ), despite a previous report to the contrary in drug-naive rats (Izquierdo et al, 2001). As novelty was most robust at the start of novel context exposure and ERK1/2 phosphorylation typically peaks $\sim 15-30 \mathrm{~min}$ following induction (Valjent et al, 2000), novelty-induced ERK activation possibly dissipated by the time of tissue collection. Remarkably, U0126 selectively attenuated ERK1/2 phosphorylation after exposure to the cocaine-paired context (Figure $2 b$ ) without an overt effect on basal ERK1/2 phosphorylation (Figure 2d). Although the latter may have been due to a floor effect or alteration in ERK1/2 phosphorylation kinetics following cocaine experience (Berhow et al, 1996), these findings support the importance of ERK1/2 activation in the restabilization of contextual cocaine memories in an instrumental setting.

Importantly, U0126 administration did not exert a protracted inhibitory effect on instrumental motor performance or on ERK1/2 activation. Specifically, it did not alter active lever responding in the extinction context (Figure 1c) or inactive lever responding in either context (Figure $1 c^{\prime}$ ) at test. Furthermore, it failed to alter ERK1/2 activation in the BLA $\sim 72 \mathrm{~h}$ later at test (Figure $2 \mathrm{f}$ ), consistent with the short half-life of U0126 ( 2 h; London and Clayton, 2008). These findings support that U0126 transiently interfered with ERK activation during memory reconsolidation.

Finally, the effects of U0126 were anatomically selective to the BLA, given that intra-BLA administration of U0126 failed to reduce ERK1/2 phosphorylation in the posterior caudate putamen $(\mathrm{pCPu})$, a brain region that is dorsally adjacent to the BLA, thus the most probable recipient of inadvertent drug diffusion (Figure 2e). Although we did not similarly examine the possible role of the central nucleus 
(CeA), a brain region dorsal-medially adjacent to the BLA, in the effects of U0126 in the present study, a role for the $\mathrm{CeA}$ in drug-memory reconsolidation has been examined repeatedly and was not supported (Wang et al, 2008; Li et al, 2010; Wu et al, 2011). ERK1/2 activation in the CeA, but not the BLA, is necessary for the incubation phenomenon, a reliable enhancement in cue-induced drug seeking following an extended drug-free period ( $\mathrm{Lu}$ et al, 2005; Li et al, 2008). The development of incubation may be facilitated by the repeated retrieval and reconsolidation of drug-related associations during abstinence (NolenHoeksema et al, 2007; Wells et al, 2011). However, in light of the above incubation studies, the present findings suggest that ERK1/2 activation is differentially involved in drugmemory reconsolidation and in the retrieval/utilization of repeatedly reconsolidated drug memories. Thus, overall, the most parsimonious interpretation of the present findings is that U0126 in the BLA interfered with ERK phosphorylation related to context-response-cocaine memory reconsolidation, per se, and thereby disrupted the subsequent influence of this associative memory on cocaine-seeking behavior.

The present findings significantly contribute to prior research, indicating that the BLA is a primary site for memory reconsolidation (Tronson and Taylor, 2007; Milton and Everitt, 2010; but see Cammarota et al, 2004) and expand upon previous findings from our laboratory, demonstrating that the BLA is a locus for the protein synthesis-dependent restabilization of instrumental, context-response-cocaine memories (Fuchs et al, 2009). ERK signaling may be key to memory reconsolidation as a regulator of gene transcription and protein synthesis via the post-translational modification of transcription factors, including Elk1 and/or CREB (Miller and Marshall, 2005). As ERK is a fundamental component of several intracellular signaling cascades (Adams and Sweatt, 2002), future research will be necessary to characterize the molecular pathways through which it mediates cocaine-memory reconsolidation.

\section{The NACc and the Reconsolidation of Cocaine Memories}

ERK inhibition in, or global neural inactivation of, the NACc following cocaine-memory reactivation failed to impair subsequent drug context-induced cocaine seeking (Figure $3 \mathrm{c}$ and $\mathrm{d}$ ). These findings were unanticipated because ERK signaling and zif268 immediate-early gene expression within the NACc have critical roles in the reconsolidation of Pavlovian context-cocaine memories required for cocaine-CPP (Miller and Marshall, 2005 and Théberge et al, 2010, respectively). The negative findings in the present study cannot be attributed to insufficient dosing, as the doses of U0126 and B + M used impair the reconsolidation of Pavlovian cocaine memories (Miller and Marshall, 2005) and the expression of drug context-induced cocaine seeking (Fuchs et al, 2008a), respectively. However, these null effects could reflect NACc-specific boundary conditions in memory reconsolidation. CPP studies typically involve a shorter timeline and fewer CS-US pairings than those in the present study (Miller and Marshall, 2005; Valjent et al, 2006; Théberge et al, 2010; Brown et al, 2008), and memory age and strength are inversely related to the ease of memory destabilization and/or disruption (Milekic and Alberini, 2002; Suzuki et al, 2004; Finnie and Nader, 2012), perhaps because remote, well-trained memories are incorporated into rigid or extensive neuronal networks (McClelland et al, 1995; Frankland and Bontempi, 2005). Thus, our negative findings may suggest either a timelimited involvement of the NACc or the resilience of NACcdependent context-response-cocaine memory traces to destabilization, perhaps due to cocaine-induced metaplasticity (Lu et al, 2006; Kalivas and O'Brien, 2008; Finnie and Nader, 2012). However, it is also possible that the NACc is not critical for reconsolidating complex, instrumental context-response-cocaine memories. Consistent with this, re-exposure to a food-paired context fails to increase ERK2 activation in the NACc concomitant with food-seeking behavior (Shiflett et al, 2008). PKM- and PKC-zeta inhibition in the NACc, following re-exposure to a remifentanil-associated runway apparatus, fails to alter the speed of approach to the drug-paired goal box, which is considered a quasi-instrumental response (Crespo et al, 2012). However, it impairs the time spent in the goal box, which is considered a measure of Pavlovian conditioning. Similarly, zif268 knockdown in the NACc disrupts the reconsolidation of a Pavlovian cocaine-memory, but not a CS-cocaine memory required for the acquisition of a new instrumental response (Théberge et al, 2010).

\section{General Conclusion}

The present study and others (Théberge et al, 2010) suggest that the NACc and BLA mediate cocaine-memory reconsolidation under different experimental conditions. The NACc is critical in the restabilization of Pavlovian cocaine memories (Miller and Marshall, 2005; Théberge et al, 2010; Crespo et al, 2012), whereas the BLA has a more global role in the reconsolidation of Pavlovian and instrumental cocaine memories (Théberge et al, 2010; Sanchez et al, 2010; Fuchs et al, 2009), at least in part through ERK-dependent processes. Future studies will be needed to parametrically vary the extent of cocaine exposure and instrumental conditioning, as well as the length of the training-to-reactivation interval, in order to gain further insight into the relative contributions of the NACc and the BLA to the restabilization of maladaptive cocaine memories. Nevertheless, the present findings support the idea that the treatment of cocaine addiction will benefit from the development of highly selective memory reconsolidation inhibitor treatments designed to assuage the impact of environmental contexts on cocaine craving.

\section{ACKNOWLEDGEMENTS}

We thank Trey Newsome and Christopher Hanlin for outstanding technical assistance and Dr Courtney Miller for providing important protocols. This work was supported by NIDA R01 DA017673, DA025646, T32 DA007244, and F31 DA028051.

\section{DISCLOSURE}

The authors declare no conflicts of interest. 


\section{REFERENCES}

Adams JP, Sweatt JD (2002). Molecular psychology: roles for the ERK MAP kinase cascade in memory. Annu Rev Pharmacol Toxicol 42: 135-163.

Berhow MT, Hiroi N, Nestler EJ (1996). Regulation of the ERK (extracellular signal regulated kinase), part of the neurotrophin signal transduction cascade, in the rat mesolimbic dopamine system by chronic exposure to morphine or cocaine. J Neurosci 16: $4707-4715$.

Brown TE, Lee BR, Sorg BA (2008). The NMDA antagonist MK-801 disrupts reconsolidation of a cocaine-associated memory for conditioned place preference but not for self-administration in rats. Learn Mem 15: 857-865.

Cammarota M, Bevilaqua LRM, Medina JH, Izquierdo I (2004). Retrieval does not induce reconsolidation of inhibitory avoidance memory. Learn Mem 11: 572-578.

Childress A, Ehrman R, McLellan AT, O’Brien C (1988). Conditioned craving and arousal in cocaine addiction: $\mathrm{A}$ preliminary report. NIDA Res Monogr 81: 74-80.

Choe ES, McGinty JF (2001). Cyclic AMP and mitogenactivated protein kinases are required for glutamate-dependent cyclic AMP response element binding protein and Elk-1 phosphorylation in the dorsal striatum in vivo. J Neurochem 75: 401-412.

Crespo JA, Stöckl P, Ueberall F, Jenny M, Saria A, Zernig G (2012). Activation of PCKzeta and PKMzeta in the nucleus accumbens core is necessary for the retrieval, consolidation, and reconsolidation of a drug memory. PLoS One 7: 1-9.

Crombag HS, Bossert JM, Koya F, Shaham Y (2008). Contextinduced relapse to drug seeking: A review. Philos Trans $R$ Soc Lond B Biol Sci 363: 3233-3243.

Derkinderen P, Enslen H, Girault JA (1999). The ERK/MAP-kinase cascade in the nervous system. NeuroReport 10: R24-R34.

Duvarci S, Nader K, LeDoux JE (2005). Activation of extracellular signal-regulated kinase- mitogen-activated protein kinase cascade in the amygdala is required for memory reconsolidation of auditory fear conditioning. Eur J Neurosci 21: 283-289.

Finnie PS, Nader K (2012). The role of metaplasticity mechanisms in regulating memory destabilization and reconsolidation. Neurosci Biobehav Rev 36: 1667-1707.

Frankland PW, Bontempi B (2005). The organization of recent and remote memories. Nat Rev Neurosci 6: 119-130.

Fricks-Gleason AN, Marshall JF (2008). Post-retrieval betaadrenergic receptor blockade: effects on extinction and reconsolidation of cocaine-cue memories. Learn Mem 15: 643-648.

Fuchs RA, Bell GH, Ramirez DR, Eaddy JL, Su Z-I (2009). Basolateral amygdala involvement in memory reconsolidation processes that facilitate drug context-induced cocaine seeking. Eur J Neurosci 30: 889-900.

Fuchs RA, Lasseter HC, Ramirez DR, Xie X (2008b). Relapse to drug seeking following prolonged abstinence: the role of environmental stimuli. Drug Discov Today Dis Models 5: 251-258.

Fuchs RA, Ramirez DR, Bell GH (2008a). Nucleus accumbens shell and core involvement in drug context-induced reinstatement of cocaine seeking in rats. Psychopharmacology (Berl) 200: 545-556.

Girault JA, Valjent E, Caboche J, Hervé D (2007). ERK2: a logical AND gate critical for drug-induced plasticity? Curr Opin Pharmacol 7: 77-85.

Goodman RH (1990). Regulation of neuropeptide gene expression. Annu Rev Neurosci 13: 111-127.

Impey S, Obrietan K, Storm DR (1999). Making new connections: role of ERK/MAP kinase signaling in neuronal plasticity. Neuron 23: $11-14$

Izquierdo LA, Viola H, Barros DM, Alonso M, Vianna MR, Furman $M$ et al (2001). Novelty enhances retrieval: molecular mechanisms involved in rat hippocampus. Eur J Neurosci 13: 1464-1467.
Kalivas PW, Obrien C (2008). Drug addiction as a pathology of staged neuroplasticity. Neuropsychopharmacology (Berl) 33: 166-180.

Kelly A, Laroche S, Davis S (2003). Activation of mitogen-activated protein kinase/extracellular signal-regulated kinase in hippocampal circuitry is required for consolidation and reconsolidation of recognition memory. J Neurosci 23: 5354-5360.

Li F, Xue Y, Wang J, Fang Q, Li Y, Zhu W et al (2010). Basolateral amygdala cdk5 activity mediates consolidation and reconsolidation of memories for cocaine cues. J Neurosci 30: 10351-10359.

Li YQ, Li FQ, Wang XY, Wu P, Zhao M, Xu CM et al (2008). Central amygdala extracellular signal-regulated kinase signaling pathway is critical to incubation of opiate craving. J Neurosci 28: 13248-13257.

London SE, Clayton DF (2008). Functional identification of a sensory mechanism required for developmental song learning. Nat Neurosci 11: 579-586.

Lu L, Hope BT, Dempsey J, Liu SY, Bossert JM, Shaham Y (2005). Central amygdala ERK signaling pathway is critical to incubation of cocaine craving. Nat Neurosci 8: 212-219.

Lu L, Koya E, Zhai H, Hope BT, Shaham Y (2006). Role of ERK in cocaine addiction. Trends Neurosci 29: 696-703.

Martin JH, Ghez C (1999). Pharmacological inactivation in the analysis of the central control of movement. J Neurosci Methods 86: 145-159.

Mazzucchelli C, Vantaggiato C, Ciamei A, Fasano S, Pakhotin P, Krezel W et al (2002). Knockout of ERK1 MAP kinase enhances synaptic plasticity in the striatum and facilitates striatalmediated learning and memory. Neuron 34: 807-820.

McClelland JL, McNaughton BL, O'Reilly RC (1995). Why there are complementary learning systems in the hippocampus and neocortex: insights from the successes and failures of connectionist models of learning and memory. Psychol Rev 102: 419-457.

Milekic MH, Alberini CM (2002). Temporally graded requirement for protein synthesis following memory reactivation. Neuron 36: 521-525.

Miller CA, Marshall JF (2005). Molecular substrates for retrieval and reconsolidation of a cocaine-associated contextual memory. Neuron 47: 873-884.

Milton AL, Everitt BJ (2010). The psychological and neurochemical mechanisms of drug memory reconsolidation: Implications for the treatment of addiction. Eur J Neurosci 31: 2308-2319.

Milton AL, Lee JL, Butler VJ, Gardner R, Everitt BJ (2008a). Intraamygdala and systemic antagonism of NMDA receptors prevents the reconsolidation of drug-associated memory and impairs subsequently both novel and previously acquired drug-seeking behaviors. J Neurosci 28: 8230-8237.

Milton AL, Lee JL, Everitt BJ (2008b). Reconsolidation of appetitive memories for both natural and drug reinforcement is dependent on beta-adrenergic receptors. Learn Mem 15: 88-92.

Nader K, Schafe GE, LeDoux JE (2000b). The labile nature of the consolidation theory. Nat Rev Neurosci 1: 216-219.

Nolen-Hoeksema S, Stice E, Wade E, Bohon C (2007). Reciprocal relations between rumination and bulimic, substance abuse, and depressive symptoms in female adolescents. J Abnormal Psych 116: 198-207.

Paxinos G, Watson C (1997). The Rat Brain in Stereotaxic Coordinates, Compact. 3rd edn. Academic Press: San Diego, CA.

Pedreira ME, Maldonado H (2003). Protein synthesis subserves reconsolidation or extinction depending on reminder duration. Neuron 38: 863-869.

Sanchez H, Quinn JJ, Torregrossa MM, Taylor JR (2010). Reconsolidation of a cocaine-associated stimulus requires amygdalar protein kinase A. J Neurosci 30: 4401-4407.

Sgambato V, Pagès C, Rogard M, Besson MJ, Caboche J (1998). Extracellular signal-regulated kinase (ERK) controls immediate 
early gene induction on corticostriatal stimulation. J Neurosci 18: 8814-8825.

Shiflett MW, Martini RP, Mauna JC, Foster RL, Peet E, Thiels E (2008). Cue-elicited reward-seeking requires extracellular signalregulated kinase activation in the nucleus accumbens. J Neurosci 28: 1434-1443.

Silingardi D, Angelucci A, De Pasquale R, Borsotti M, Squitieri G, Brambilla $\mathrm{R}$ et al (2011). ERK pathway activation bidirectionally affects visual recognition memory and synaptic plasticity in the perirhinal cortex. Front Behav Neurosci 5: 84.

Sorg BA (2012). Reconsolidation of drug memories. Neurosci Biobehav Rev 36: 1400-1417.

Suzuki A, Josselyn SA, Frankland PW, Masushige S, Silva AJ, Kida S (2004). Memory reconsolidation and extinction have distinct temporal and biochemical signatures. J Neurosci 24: 4787-4795.

Sweatt JD (2001). The neuronal MAP kinase cascade: a biochemical signal integration system subserving synaptic plasticity and memory. J Neurochem 76: 1-10.

Théberge FR, Milton AL, Belin D, Lee JL, Everitt BJ (2010). The basolateral amygdala and nucleus accumbens core mediate dissociable aspects of drug memory reconsolidation. Learn Mem 17: 444-453.

Tronson NC, Taylor JR (2007). Molecular mechanisms of memory reconsolidation. Nat Rev Neurosci 8: 262-275.

Valjent E, Corbillé AG, Bertran-Gonzalez J, Hervé D, Girault JA (2006). Inhibition of ERK pathway or protein synthesis during reexposure to drugs of abuse erases previously learned place preference. Proc Natl Acad Sci USA 21: 2932-2937.

Valjent E, Corvol J-C, Pagès C, Besson M-J, Maldonado R, Caboche $\mathrm{J}$ (2000). Involvement of the extracellular signal-regulated kinase cascade for cocaine-rewarding properties. I Neurosci 20: 8701-8709.

Wang XY, Zhao M, Ghitza UE, Li YQ, Lu L (2008). Stress impairs reconsolidation of drug memory via glucocorticoid receptors in the basolateral amygdala. J Neurosci 28: 5602-5610.

Wells AM, Lasseter HC, Xie X, Cowhey KE, Reittinger AM, Fuchs RA (2011). Interaction between the basolateral amygdala and dorsal hippocampus is critical for cocaine memory reconsolidation and subsequent durg context-induced cocaine-seeking behavior in rats. Learn Mem 18: 693-702.

Wu P, Xue YX, Ding ZB, Xue LF, Xu CM, Lu L (2011). Glycogen synthase kinase $3 \beta$ in the basolateral amygdala is critical for the reconsolidation of cocaine reward memory. J Neurochem 118: 113-125.

Zhai H, Li Y, Wang X, Lu L (2008). Drug-induced alterations in the extracellular signal-regulated kinase (ERK) signaling pathway: implications for reinforcement and reinstatement. Cell $\mathrm{Mol}$ Neurobiol 28: 157-172.

Zhang L, Lou D, Jiao H, Zhang D, Wang X, Xia Y et al (2004). Cocaine-induced intracellular signaling and gene expression are oppositely regulated by the dopamine D1 and D3 receptors. J Neurosci 24: 3344-3354.

Supplementary Information accompanies the paper on the Neuropsychopharmacology website (http://www.nature.com/npp) 